\title{
Divertor currents optimization procedure for JET-ILW high flux expansion experiments
}

\author{
G. Calabrò $^{\mathrm{a}}$, F. Maviglia ${ }^{\mathrm{b}}$, S. Minucci ${ }^{\mathrm{a}, *}$, B. Viola $^{\mathrm{c}}$, JET Contributors ${ }^{1}$ \\ ${ }^{\text {a }}$ Department of Economics, Engineering, Society and Business Organization (DEIm), University of Tuscia, Largo dell'Università snc, 01100 Viterbo, Italy \\ b Consorzio CREATE, Via Claudio 21, 80125, Napoli, Italy \\ ${ }^{\mathrm{c}}$ ENEA for EUROfusion, Via E. Fermi 45, 00044 Frascati, Roma, Italy
}

\section{A R T I C L E I N F O}

\section{Keywords:}

Advanced configuration

Quadratic programming optimization

Flux expansion

Scrape-off layer

\begin{abstract}
A B S T R A C T
This paper deals with a divertor coil currents optimized procedure to design High Flux Expansion (HFE) configurations in the JET tokamak aimed to study the effects of flux expansion variation on the radiation fraction and radiated power re-distribution. A number of benefits of HFE configuration have been experimentally demonstrated on TCV, EAST, NSTX and DIII-D tokamaks and are under investigation for next generation devices, as DEMO and DTT. The procedure proposed here exploits the linearized relation between the plasma-wall gaps and the Poloidal Field (PF) coil currents. Once the linearized model is provided by means of CREATE-NL code, the divertor coils currents are calculated using a constrained quadratic programming optimization procedure, in order to achieve HFE configuration. Flux expanded configurations have been experimentally realized both in ohmic and heated plasma with and without nitrogen seeding. Preliminary results on the effects of the flux expansion variation on total power radiation increase will be also briefly discussed.
\end{abstract}

\section{Introduction}

Heat and particle loads on the plasma facing components are among the most challenging issues to be solved to design a nuclear fusion reactor $[1,2]$. An approach to handle the heat exhaust power is to use alternative magnetic configurations, such as Snowflake Divertor (SF) [3] and recently described divertor with a strong flux flaring in a single divertor leg $[4,5]$. Such a configuration places the second x-point near the plate, causing flared field lines in that region which spread the heat load over a larger area and increase the line connection length. Recently, a number of additional benefits of High Flux Expansion (HFE) configurations have been experimentally demonstrated on TCV [6] and DIII-D [7]. On the former, HFE configuration showed deeper detachment and a reduction of the radiation location sensitivity with respect to the plasma core density, with increasing flux expansion and flaring; on the latter, it showed that increasing flux expansion and flaring allow for detachment at lower density and higher pedestal pressure. The SF configuration is characterized by a second-order null (x-point) in the poloidal magnetic field $\left(\mathrm{B}_{\mathrm{P}}\right)$, where both $\mathrm{B}_{\mathrm{P}}$ itself and its spatial derivatives vanish. This splits the separatrix in the nearness of the null into six segments: two of them enclose the confined plasma and the others lead to the machine wall (the divertor legs). The poloidal cross-section of the obtained magnetic flux surfaces with a hexagonal null-point resembles a snowflake. Theoretical studies indicate that the SF magnetic geometry may lead to both higher power losses during Scrape-Off Layer (SOL) transport and an increased plasma wetted area of the wall [8]. As it was realized in the first assessment of the SF [3], an exact SF configuration is topologically unstable: any plasma perturbation or imbalance of the Poloidal Field (PF) coils current splits the second-order null into two first-order nulls, leading to a variety of topologicallystable SF-like configurations [8]. The secondary null can be moved around to change its distance from the first one and to form a magnetic configuration that features either a contracting or a flaring geometry near the plate $[3-5,8]$. The linear dependence of the gradient of magnetic field $B_{P}$ in the primary null with respect to distance between the two nulls is described in $[9,10]$ and characterizes the interdependence of the field structures of both nulls. This feature will be analyzed for JET: the flaring of the magnetic flux (characterized by the magnetic field gradient) in the primary null is affected by the presence of the secondary null. This flaring is then directly translated in the increased wetted surface area and reduced heat flux $[5,8]$ or in a total radiated power increase, as it will be discussed in the paper.

In the past at JET, with the MkI divertor, a systematic study of the influence of $\mathrm{x}$-point height and poloidal flux expansion has been set up

\footnotetext{
* Corresponding author.

E-mail address: simone.minucci@unitus.it (S. Minucci).

${ }^{1}$ See the author list of X. Litaudon et al 2017 Nucl. Fusion 57102001.
} 
$[11,12]$ showing minor differences in the radiation distribution, whereas in [4], experiments and simulations have shown an enhancement of detachment as the flux expansion was increased. More recently at JET, equipped with ITER-like Wall [13], radiative seeded scenarios have been studied and only a maximum $75 \%$ radiation fraction has been achieved [14]. However, recent predictive studies [15] have shown that HFE configurations increase the radiation in the proximity of the x-point and have an ionization front extending further in the Scrape-Off Layer (SOL) than in the Low Flux Expansion (LFE) case. In addition, HFE cases do seem to offer a benefit in reducing the nitrogen concentration needed to obtain a given radiated power level [15]. Here, we will discuss the modelling, creation and control of HFE configurations at JET-ILW, characterized by the presence of two nearby poloidal field nulls in the divertor region, aimed to study the effects of flux expansion variation on radiation fraction and radiated power re-distribution.

The paper is organized as follows: Section 2 describes the HFE configuration design and optimization, taking into account the technological constraints of JET tokamak. In Section 3, preliminary experimental results of ohmic, nitrogen seeded high confinement $(\mathrm{H}-$ mode) HFE, and LFE discharges will be discussed, supported by interpretative 2D edge modelling. Finally, Section 4 draws the main conclusions and outlook.

\section{Constrained optimization procedure}

The JET tokamak has eight PF coils potentially useful for plasma shape control (see Fig. 1) and are denoted by $\mathrm{P}_{1} \ldots \mathrm{P}_{4}$ and $\mathrm{D}_{1} \ldots \mathrm{D}_{4}$. The Pcoils are not equipped with their own power supply but are connected each other and fed by five circuits [10]. The currents flowing in these circuits are denoted by $I_{P 1 E}, I_{P F X}, I_{S H A}, I_{P 4 T}, I_{P 41}$, whereas each divertor coil (D-coils) is fed by its own power supply that sustain a current denoted by $I_{D i}$, with $i=1 \ldots 4$. Therefore, nine circuits are available to the

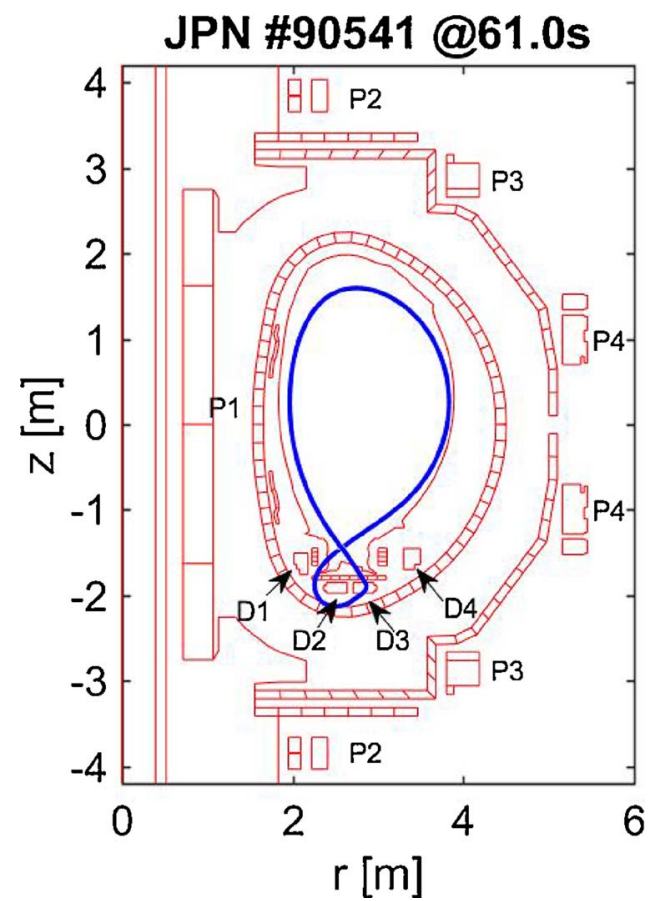

Fig. 1. Cross section of the JET tokamak. The plasma boundary of the JET discharge \#90541 at $61 \mathrm{~s}$ is shown in blue. The poloidal coils (P1-P4 and D1-D4) and the toroidal coils, which surround the plasma ring, produce the required magnetic field for plasma confinement. Here, the D coils current are used in the optimization procedure in order to locally modify the magnetic topology in the divertor region.(For interpretation of the references to colour in this figure legend, the reader is referred to the web version of this article.) plasma control system. The circuit $\mathrm{P}_{1 \mathrm{E}}$ is used to control the plasma current, whereas the other eight circuits are used to control the plasma shape.

The magnetic geometry in the divertor can be modified by changing the flux expansion at the target $\mathrm{f}_{\mathrm{x}, \mathrm{t}}=\mathrm{dr}_{\text {target }} / \lambda_{\mathrm{q}}: \mathrm{dr}_{\text {target }}$ is the distance along the First Wall boundary between the inner (or outer) strike points of the separatrix and of the SOL boundary (i.e. the flux surface at one efolding length power decay $\lambda_{\mathrm{q}}$ away from the separatrix); $\lambda_{\mathrm{q}}$ is the SOL width on the outer plane containing the plasma centroid. It should be noted that in JET, because of the geometry of the target plates, the distance between the inner (or outer) strike points of SOL boundary and of the separatrix along the First Wall might be very high even though their Euclidean distance on the poloidal plane is not. In order to avoid this issue we consider the Full Flux Expansion at the target [16] as the ratio between $\mathrm{dr}_{\text {target,tube }}$ (i.e. the poloidal expansion of the magnetic flux tube at the target) and the SOL width (see Fig. 2a in Section 3). In addition, we consider the flux expansion at x-point as $\mathrm{f}_{\mathrm{x}, \mathrm{xp}}=\mathrm{dr}_{\mathrm{xpoint}} /$ $\lambda_{\mathrm{q}}$, where $\mathrm{dr}_{\mathrm{xpoint}}$ is the Euclidian distance between the x-point and the SOL boundary along the horizontal line inward and outward the $\mathrm{x}$-point height (see Fig. 2a). For sake of consistency, the same rectangular grid has been used when scanning the SOL width on the plane containing the plasma centroid. Specifically, SOL width $\lambda_{\mathrm{q}}$ of 2,5 and $10 \mathrm{~mm}$ have been considered. Here, HFE configuration characterized by the presence of 2-nearby divertor poloidal field nulls have been designed and optimized by means of CREATE-NL code (non-linear plasma evolution code), described in [17]. The procedure proposed for the design and optimization of the equilibria using the CREATE-NL code exploits the linearized relation between the plasma-wall gaps (the distance between the plasma surface and the first wall, measured along a given direction [18]) and the PF currents in two steps. The first step allows to have a first cut of the HFE equilibrium starting from a standard Single Null (SN) LFE configuration; a new equilibrium with a second null point within a limited distance from the LFE x-point is obtained, forcing the plasma boundary to be almost unchanged, apart from the region in the nearness of the null point. The second step refines the plasma shape and possibly reduces the PF coil currents while fulfilling the machine technological constraints. Once the linearized model is provided by means of CREATE-NL code, the divertor coils current needed to achieve the HFE configuration are calculated by means of a constrained quadratic programming optimization procedure $[19,20]$, generally stated as follows:

$\min _{\underline{x}} \frac{1}{2} \underline{x}^{T} \underline{\underline{H}} \underline{x}+\underline{f}^{T} \underline{x}$ subject to: $\left\{\begin{array}{l}\underline{\underline{A}} \cdot \underline{x}=\underline{b} \\ \underline{l_{b}} \leq \underline{x} \leq \underline{u}_{b}\end{array}\right.$

where the symmetric matrix $\underline{\underline{H}}$ represents the quadratic term, the vector $\underline{f}$ is the linear term, whilst the matrix $\underline{\underline{A}}$ and the vector $\underline{b}$ represent respectively the linear coefficients and the constant in the constraint of Eq. (1). At last, vectors $\underline{l}_{b}$ and $\underline{u}_{b}$ represent respectively the lower and upper bounds elementwise in (1). Once matrix $\underline{\underline{H}}$ and vector $\underline{f}$ are set up respectively as identity matrix and zero vector, finding a minimum for a problem specified by Eq. (1) turns into minimizing the Euclidean norm of the unknown vector $\underline{x}$ and guaranteeing the convexity of the objective function as well as the uniqueness of the optimization problem solution. Hence, Eq. (1) is adapted to the specific problem of achieving HFE plasma configurations while minimizing currents variation $\underline{\Delta I}$ in the PF coils subject to technological restrictions. Indeed, the vector $\underline{x}=\underline{\Delta I}$ must accomplish specific constraints, modelled by means of the linear equality constraints set thanks to the adopted linearized model: plasma shape and total plasma current are requested to be kept unchanged, whilst flux expansion is increased. Moreover $\underline{x}$ must be bounded according to the current restrictions on PF coil power supplies. Consequently, the constrained quadratic programming optimization procedure turns into the following problem: 


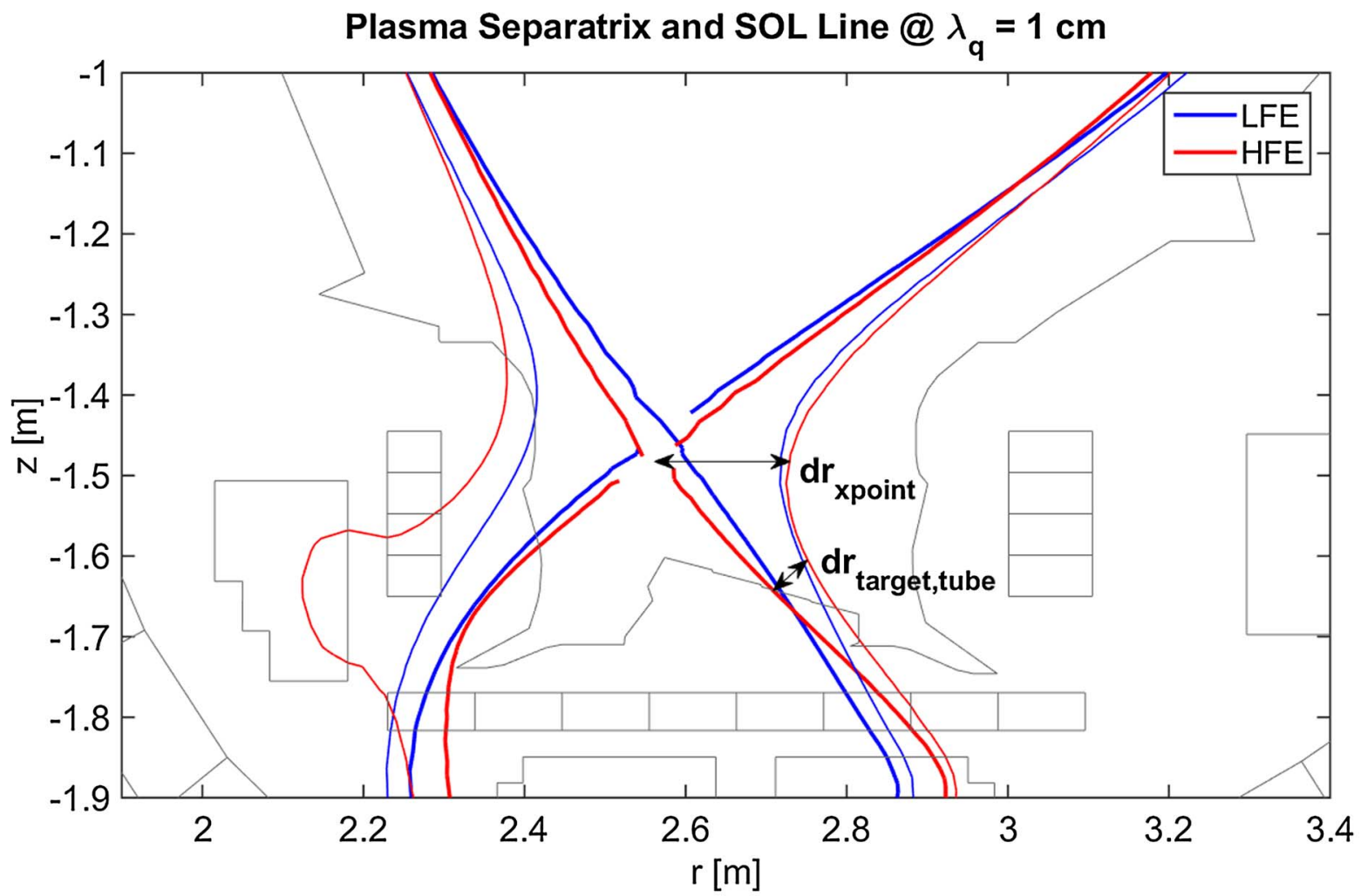

a)

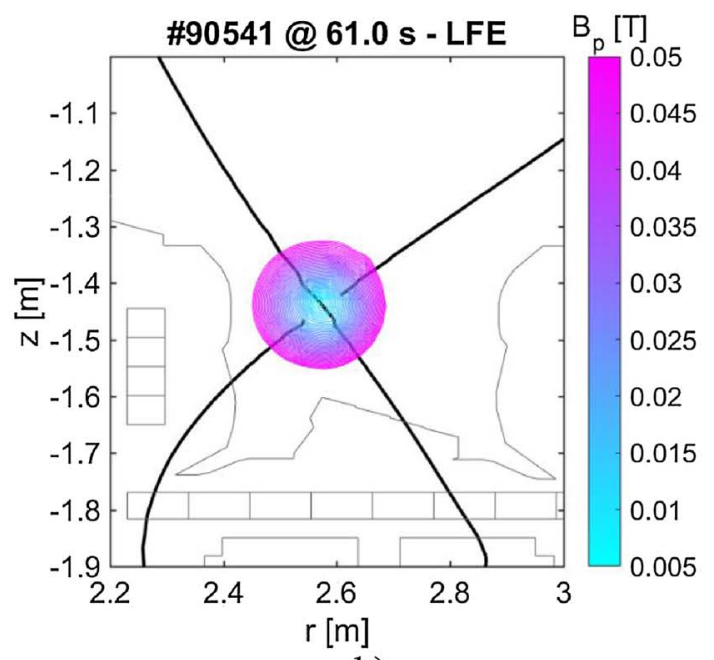

b)

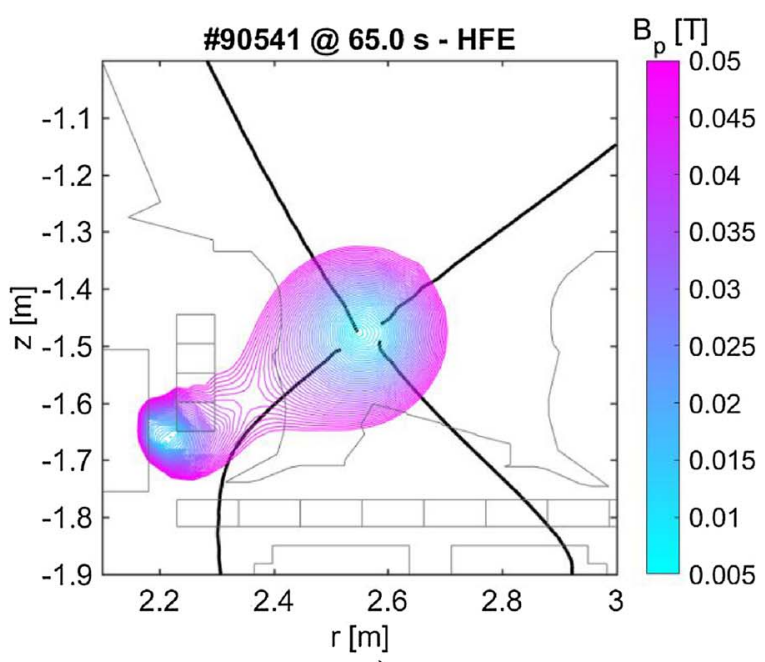

c)

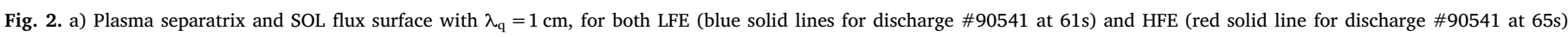

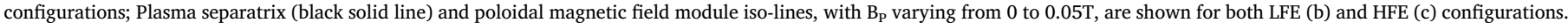
(For interpretation of the references to colour in this figure legend, the reader is referred to the web version of this article.)

$\min \|\underline{\Delta I}\|_{2}$ subject to: $\left\{\begin{array}{l}\underline{A}_{\text {gaps }-I} \cdot \underline{\Delta I}=\underline{0} \\ \underline{L}_{\text {plasma }-I}^{T} \cdot \underline{\Delta I}=\underline{0} \\ \underline{A}_{\text {FExp-I } I} \cdot \underline{\Delta I}=\underline{\delta}_{F E x p} \\ \underline{I}_{\min }-\underline{I}_{0} \leq \underline{\Delta I} \leq \underline{I}_{\max }-\underline{I}_{0}\end{array}\right.$

where:

- $\underline{\underline{A}}_{\text {gaps-I }}$ represents the linearized relationship between the $\mathrm{I}_{\mathrm{PF}}$ currents and the plasma-wall gaps to be controlled (the first constraint states that the variation of $\mathrm{I}_{\mathrm{PF}}$ currents does not affect the plasmawall gaps); the following five gaps have been specifically considered [17] in our analyses: Radial Outer Gap (ROG), Radial Inner Gap
(RIG), Top Gap (TOG), R coordinate of Outer Strike Point (RSOGB) and Z coordinate of Inner Strike Point (ZSIGB);

- $\underline{L}_{\text {plasma-I }}$ represents the mutual inductance vector between the plasma and the active coils (the second constraint states that the variation of $\mathrm{I}_{\mathrm{PF}}$ currents does not affect the total plasma current);

- $\underline{\underline{A}}_{F E x p-I}$ represents the linearized relationship between the $\mathrm{I}_{\mathrm{PF}}$ currents and the magnetic flux expansion (the third constraint states that the variation of $\mathrm{I}_{\mathrm{PF}}$ currents changes the flux expansion, here assumed to be the Full flux Expansion $\mathrm{f}_{\mathrm{x}, \mathrm{t}}$ of the SOL boundary with decay length $\lambda_{\mathrm{q}}=2 \mathrm{~mm}$, according to $\underline{\delta}_{F E x p}$ ).

- $\underline{I}_{0}$ is the $\mathrm{I}_{\mathrm{PF}}$ currents vector in the reference equilibrium (the last constraint bounds the currents flowing into the $\mathrm{I}_{\mathrm{PF}}$ currents 
Table 1

Flux expansion on inner and outer x-point plane and targets for JET-ILW LFE and HFE discharges. A SOL width of $\lambda \mathrm{q}=2 \mathrm{~mm}$ has been considered, accordingly with the lengthdecay of the thermal power in the SOL in JET tokamak [2].

\begin{tabular}{lllll}
\hline & $\mathrm{f}_{\mathrm{x}, \mathrm{xp} / \mathrm{IN}}$ & $\mathrm{f}_{\mathrm{x}, \mathrm{xp} / \mathrm{OUT}}$ & $\mathrm{f}_{\mathrm{x}, \mathrm{t} / \mathrm{IN}}$ & $\mathrm{f}_{\mathrm{x}, \mathrm{t} / \mathrm{OUT}}$ \\
\hline $\mathrm{HFE}$ & 42.52 & 40.44 & 12.63 & 5.96 \\
$\mathrm{JPN} \# 90541 @ 65 \mathrm{~s}$ & & & & \\
$\mathrm{I}_{\mathrm{D} 1}=6.36 \mathrm{kA}, \mathrm{I}_{\mathrm{D} 2}=5.04 \mathrm{kA}, \mathrm{I}_{\mathrm{D} 3}=11.19$ & & & & \\
$\quad \mathrm{kA}, \mathrm{I}_{\mathrm{D} 4}=0 \mathrm{kA}$ & 35.66 & 34.39 & 6.20 & 4.08 \\
$\mathrm{LFE}$ & & & & \\
$\mathrm{JPN} \# 90541 @ 61 \mathrm{~s}$ & & & & \\
$\mathrm{I}_{\mathrm{D} 1}=1.51 \mathrm{kA}, \mathrm{I}_{\mathrm{D} 2}=13.91 \mathrm{kA}, \mathrm{I}_{\mathrm{D} 3}=6.41$ & & & & \\
$\quad \mathrm{kA}, \mathrm{I}_{\mathrm{D} 4}=-4.34 \mathrm{kA}$ & & & & \\
\end{tabular}

according to the technological restrictions of each power supply, in terms of minimum and maximum attainable values; specifically, the following lower and upper bounds have been considered for each of the divertor coil: $I_{\mathrm{D} 1}\{0,19 \mathrm{kA}\}, \quad \mathrm{I}_{\mathrm{D} 2}\{0,37 \mathrm{kA}\}, \quad \mathrm{I}_{\mathrm{D} 3}\{0,37 \mathrm{kA}\}$, $\mathrm{I}_{\mathrm{D} 4}\{-18 \mathrm{kA}, 0\}$.

It should be noted that the upper and lower current bounds are additionally constrained by the power supplies topology and in particular by the AC-DC converters [17]. In particular, they are two voltage quadrants rectifiers, able to change the polarity of the only voltage across their terminals, whilst the current polarity can be changed only by means of a switch that inverts the taps of the coils. For this reason, the poloidal currents can range only in one quadrant of the currentvoltage operating plane, adding an additional constraint for the optimization problem. In the experiments discussed hereafter, the maximum attainable flux expansion has been mainly limited by the polarity of the D-coils (positive for $I_{D 1}, I_{D 2}, I_{D 3}$ and negative for $I_{D 4}$ ) and in particular by that of D4 coil. As discussed in [21], an increase of the flux expansion could be achieved by changing the polarity on D2 and D4 coils.

\section{Experimental results}

Initial HFE ohmic and nitrogen seeded H-mode discharges, at plasma current $\mathrm{I}_{\mathrm{P}}=1.8 \mathrm{MA}$ have been successfully achieved at JET ILW. Table 1 reports the values of both $\mathrm{f}_{\mathrm{x}, \mathrm{t}}$ and $\mathrm{f}_{\mathrm{x}, \mathrm{xp}}$, considering a SOL width of $\lambda_{\mathrm{q}}=2 \mathrm{~mm}$, for the ohmic discharge \#90541 when moving from LFE to HFE configuration. Plasma separatrix and flux surface at $\lambda_{\mathrm{q}}=1 \mathrm{~cm}$ for both LFE and HFE configurations are shown in Fig. 2a. Plasma separatrix and poloidal magnetic field module iso-lines, with $\mathrm{B}_{\mathrm{P}}$ varying from 0 to $0.05 \mathrm{~T}$, are shown for both LFE (Fig. 2b) and HFE (Fig. 2c) configurations. It should be noted that the magnetic field flatness region is increased for HFE configuration thanks to the presence of a second x-point.

An experimental flux expansion increase by a factor of $\sim 20 \%$ at primary x-point inwards and $\sim 50 \%$ on the outer divertor target has been achieved, thanks to the generation of a second null point close to inner part of the first wall. Fig. 3 shows a comparison between experimental LFE (blue color) and HFE configuration (red color) in terms of plasma-wall gaps (i.e. ROG, RIG, TOG, RSOGB and ZSIGB), plasma current $I_{P}$ and divertor coils current needed to increase the flux expansion. The distance between the two null-points, i.e the proximity to the exact SF $[9,10]$, is parametrized here by the dimensionless factor $\sigma=\mathrm{D} / \mathrm{a}$, where $\mathrm{D}$ is the null-points separation distance and $\mathrm{a}$ is the plasma minor radius. SF like configuration corresponds to $\sigma$ close to zero. The mutual position of the primary and secondary null-points determines the local geometry of the null region and hence the properties of the divertor. Experimental values of the flux expansion at the inner and outer $x$-point $f_{x, x p}$ vs. SF proximity parameter $\sigma$ are shown in Fig. 4 for JET-ILW discharges. Both inner and outer $\mathrm{f}_{\mathrm{x}, \mathrm{xp}}$ increase when moving from LFE $(\sigma=1)$ to HFE configuration $(0.25<\sigma<0.45)$. The maximum flux expansion increase has been obtained for the discharge \#92086 by relaxing the plasma distance to the inner wall. It should be noted that this configuration cannot be used in H-mode discharges because of the limitations on the permitted operational plasma-wall distance for heated plasmas.

Finally, the aforementioned LFE and HFE configurations have been recently used to address the physics of a possible dependence of radiative volume and total radiated power on the distance between the two nulls [15]. Aim of this study has been the evaluation of the impact of main magnetic divertor geometry parameters, as the flux expansion and the connection length, on the radiation pattern disentangled by the change of recycling happening at the same time, focusing on bolometer and Langmuir probe measurements analysis supported by EDGE2DEIRENE code [22,23] interpretative modelling. A detailed analysis of the power balance has been set up, as well discussed in [15], to

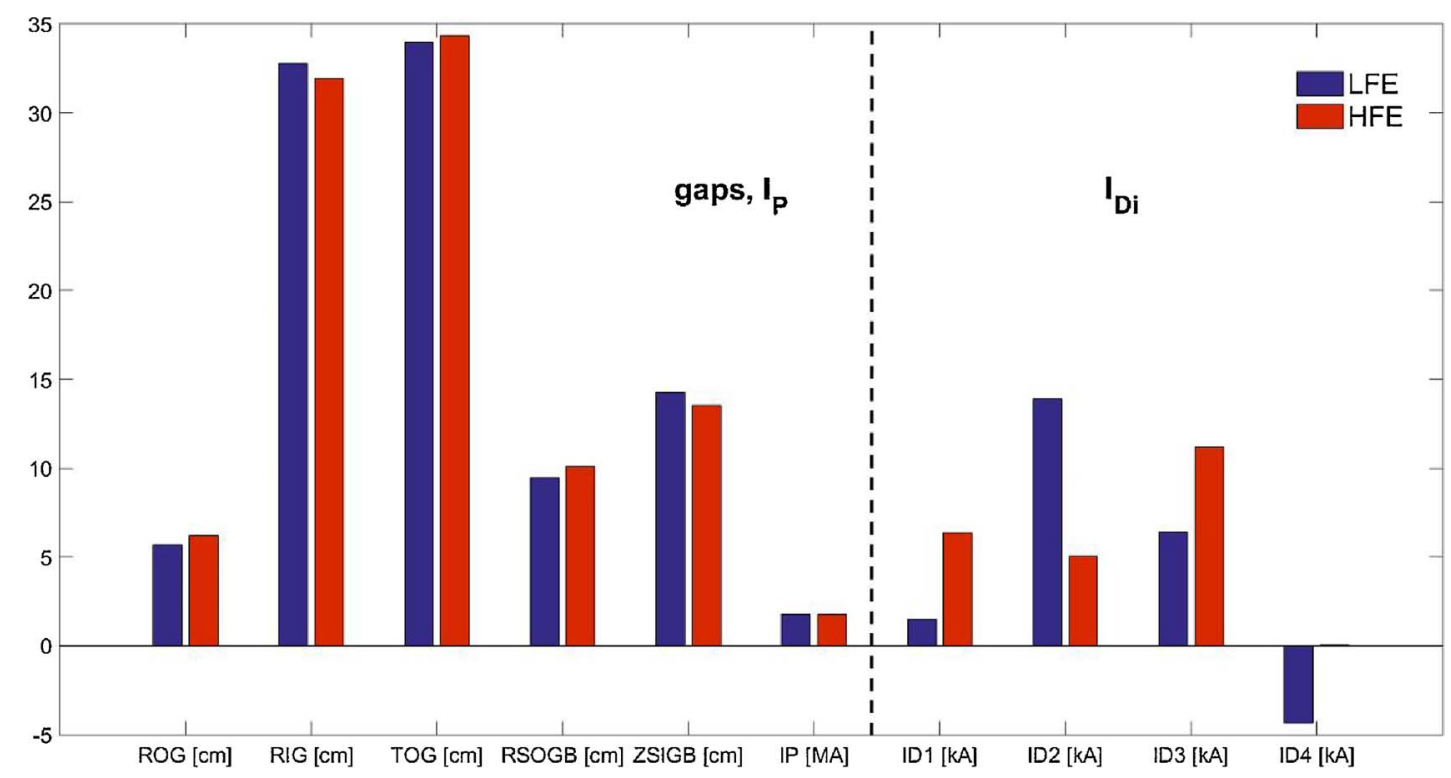

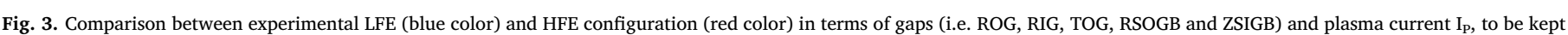

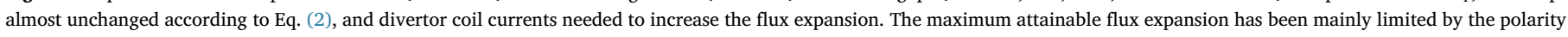
on D4 coil. (For interpretation of the references to colour in this figure legend, the reader is referred to the web version of this article.) 


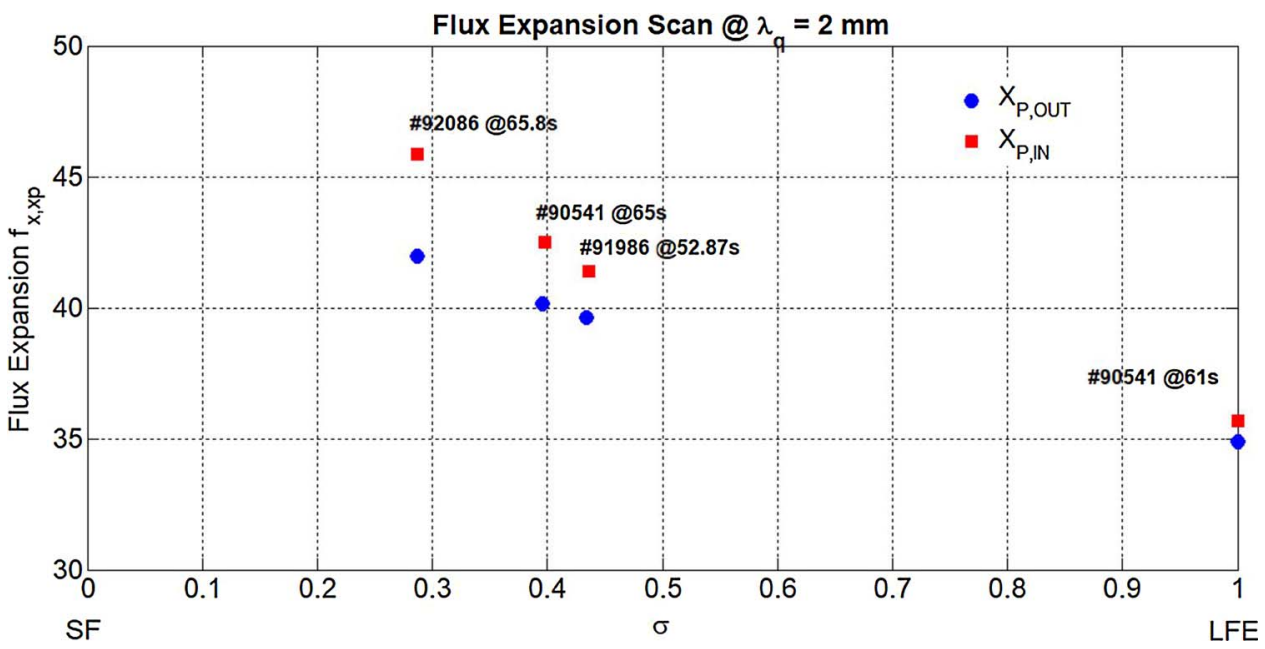

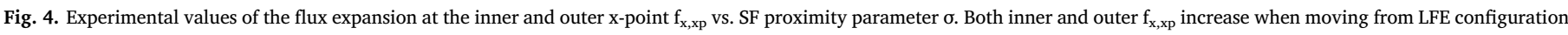
$(\sigma=1)$ to HFE configuration $(0.25<\sigma<0.45)$, characterized by the presence of 2 -nearby poloidal field nulls.

physically investigate the reason of the increase of the radiated power for HFE discharges. In summary, although the nitrogen radiation is constant in all the studied cases, the increase of $20 \%$ of the total radiation power in the high flux expansion case does mainly seem due to the molecular and charge exchange losses. As discussed in [15], an increase of charge exchange losses has been related to an increase of connection length and flux expansion both at x-point at strike points position.

\section{Conclusions}

The demonstration of the possibility of creating and controlling a two nearby poloidal field nulls in JET-ILW tokamak has been achieved, showing an increase on magnetic poloidal flux expansion both at $\mathrm{x}$ point and strike points position. Initial experiments with a second null, located in the proximity of the inner divertor region have been performed, forming a configuration with significant distance between the two nulls and a contracting geometry near the target plates, leading to an increase of the main magnetic divertor geometry parameters. In addition, preliminary nitrogen seeded H-mode experiments have been set-up showing an increase of the total radiated power of the same factor of the flux expansion increase. Further experiments will be devoted to change the divertor coils polarities in order to move the secondary null point on the outer divertor region and consequently increase the outer $\mathrm{x}$-point and target flux expansion.

\section{Acknowledgments}

The authors would like to acknowledge Prof. R. Albanese, Dr. F. Crisanti and Dr. G. Artaserse for their fruitful suggestions. This work has been carried out within the framework of the EUROfusion Consortium and has received funding from the Euratom research and training programme 2014-2018 under grant agreement No. 633053. The views and opinions expressed herein do not necessarily reflect those of the European Commission.

\section{References}

[1] A. Loarte, et al., Chapter 4: power and particle control, Nucl. Fusion 47 (6) (2007) pp. S203.

[2] Team Asdex Upgrade, T. Eich, B. Sieglin, A. Scarabosio, W. Fundamenski, R.J. Goldston, A. Herrmann, Inter-ELM power decay length for JET and ASDEX upgrade: measurement and comparison with heuristic drift-based model, Phys. Rev. Lett. 107 (21) (2011) 215001.
[3] D.D. Ryutov, Geometrical properties of a snowflake divertor, Phys. Plasmas 14 (6) (2007) 064502.

[4] R.A. Pitts, B.P. Duval, A. Loarte, J.M. Moret, J.A. Boedo, D. Coster, I. Furno, J. Horacek, A.S. Kukushkin, D. Reiter, J. Rommers, Divertor geometry effects on detachment in TCV, J. Nucl. Mater. 290-293 (2001) 940-946.

[5] M. Kotschenreuther, P. Valanju, B. Covele, S. Mahajan, Magnetic geometry and physics of advanced divertors: the X-divertor and the snowflake, Phys. Plasmas 20 (10) (2013) 102507.

[6] C. Theiler, et al., Results from recent detachment experiments in alternative divertor configurations on TCV, Nucl. Fusion 57 (7) (2017) 072008.

[7] B.M. Covele, M. Kotschenreuther, S. Mahajan, P. Valanju, A. Leonard, J. Watkins, M. Makowski, M. Fenstermacher, H. Si, Increased heat dissipation with the X-divertor geometry facilitating detachment onset at lower density in DIII-D, Nucl. Fusion 57 (8) (2017) 086017.

[8] D.D. Ryutov, M.A. Makowski, M.V. Umansky, Local properties of the magnetic field in a snowflake divertor, Plasma Phys. Control. Fusion 52 (10) (2010) 105001.

[9] D.D. Ryutov, V.A. Soukhanovskii, The snowflake divertor, Phys. Plasmas 22 (11) (2015) 110901.

[10] G. Calabrò, et al., EAST alternative magnetic configurations: modelling and first experiments, Nucl. Fusion 55 (8) (2015) 083005.

[11] C.G. Lowry, et al., Divertor configuration studies on JET, J. Nucl. Mater. 241-243 (1997) 438-443, http://dx.doi.org/10.1016/S0022-3115(97)80078-5 ISSN 00223115 .

[12] A. Loarte, et al., Plasma detachment in JET mark I divertor experiments, Nucl. Fusion 38 (3) (1998) 331-371.

[13] E. Joffrin, et al., First scenario development with the JET new ITER-like wall, Nucl. Fusion 54 (1) (2013) 013011.

[14] C. Lowry, M. Wischmeier, A. Huber, C.F. Maggi, K. McCormick, M. Reinke, et al., Impurity seeding on JET to achieve power plant like divertor conditions, Presented at 25th IAEA Fusion Energy Conference (FEC 2014), Saint Petersburg, 2014.

[15] B. Viola, G. Calabró, A.E. Jaervinen, I. Lupelli, F. Maviglia, S. Wiesen, M. Wischmeier, J.E.T. Contributors, EDGE2D-EIRENE simulations of the impact of poloidal flux expansion on the radiative divertor performance in JET, Nucl. Mater. Energy 12 (2017) 786-790.

[16] H. Reimerdes, et al., TCV experiments towards the development of a plasma exhaust solution, Nucl. Fusion 57 (12) (2017) 126007.

[17] R. Albanese, R. Ambrosino, M. Mattei, CREATE-NL + : a robust control-oriented free boundary dynamic plasma equilibrium solver, Fusion Eng. Des. 96 (2015) 664-667.

[18] M. Ariola, A. Pironti, Magnetic Control of Tokamak Plasmas, Advances in Industrial Control Vol. 187 Springer-Verlag, London, 2008.

[19] T.F. Coleman, Y. Li, A reflective Newton method for minimizing a quadratic function subject to bounds on some of the variables, SIAM J. Optim. 6 (4) (1996) 1040-1058.

[20] N. Gould, P.L. Toint, Preprocessing for quadratic programming, Math. Program. 100 (1) (2004) 95-132.

[21] G. Calabrò, et al., Divertor configuration with two nearby poloidal field nulls: modelling and experiments for EAST and JET tokamaks, Presented at 22nd International Conference on Plasma Surface Interactions in Controlled Fusion Devices (PSI 22) Rome (2016).

[22] R. Simonini, G. Corrigan, G. Radford, J. Spence, A. Taroni, Models and numerics in the multi-fluid 2-D edge plasma code EDGE2D/U, Contrib. Plasma Phys. 34 (2-3) (1994) 368-373.

[23] S. Wiesen, EDGE2D/EIRENE code interface report, Technical Report Online available: http://www.eirene.de/e2deir report 30jun06.pdf 2006 